\title{
Phylocanvas.gl: A WebGL-powered JavaScript library for large tree visualisation
}

Khalil Abudahab, Anthony Underwood, Ben Taylor, Corin Yeats, David M Aanensen

Centre for Genomic Pathogen Surveillance https://pathogensurveillance.net

phylocanvas@cgps.group

\section{Introduction}

Representing the relationships between large sets of data requires visualisations that, ideally, allow broad relationships to be seen at the global level and also fine scale relationships to be seen at the local data level. One such method utilised widely within the fields of phylogenetics and genomic epidemiology (but broadly appropriate for any data types) is to represent the closeness (or distance between) of multiple points of data as a tree. As datasets increase in size we need ever-scalable and intuitive ways to visualise and interact with these trees to help elucidate meaning from relationships depicted. Delivery of this kind of data within web applications that enhance the integration of such trees with additional data types requires the development of lightweight, browserbased JavaScript libraries which are directed towards ease of integration and utility for extensibility via the web. Projects such as Phylotree.js ${ }^{1}$, jsPhyloSVG ${ }^{2}$, and PhyD3 ${ }^{3}$ provide extensible libraries which can handle modest size trees but are not optimised for trees with hundreds of thousands of leaves. Previously we developed Phylocanvas (https://phylocanvas.org) to utilise the HTML5 canvas API (https://developer.mozilla.org/en-US/docs/Web/API/Canvas API), and to scale to several thousand leaves easily and intuitively within a library that can be reused and embedded in users applications (e.g. see uses within applications developed within team - https://microreact.org, https://pathogen.watch but also by thrid-party application such as the Canadian Infectious Disease Platform IRIDA ${ }^{4}$ (https://irida.ca), the Cereals Genomics Database ${ }^{5}$ (https://www.cerealsdb.uk.net) and Phandango ${ }^{6}$ (https://phandango.net). Additionally, packaging libraries for access through programming interfaces such as $\mathrm{R}$ (https://cran.r-project.org/ web/packages/phylocanvas/index.html and ease of delivery through NPM to enhance reuse are standard.

The limited scalability represented through uses of CANVAS within Phylocanvas, and the increasing need to visualise ever larger trees (as a specific example for viewing global phylogenies for $>$ million leaves of the SARS-CoV-2 viral genomes) has been addressed through the development of Phylocanvas.gl which utilises the WebGL library and has been enhanced to scale to hundreds of thousands of leaves. Here we detail main features, with links to code examples and use cases and describe its utility.

\section{Implementation}

Here we present a new implementation of Phylocanvas, written to incorporate WebGL (Web Graphics Library), a JavaScript API to render graphics in most modern web browsers without the use of plug-ins. WebGL allows GPU-accelerated image processing as part of the web page canvas thereby enabling Phylocanvas.gl to both render very large trees and allow interactivity via Javascript events. The primary result of this is that rendering the tree is much quicker compared to using the HTML canvas element allowing trees of $100 \mathrm{~K}+$ nodes to be displayed 
The library has been written from the ground up so that all properties of the tree are all stored in JSON. When changes are made to the tree, the underlying JSON is updated to reflect the changes. This allows a user to use this JSON as a store in which all properties of the tree are recorded.

The tree object implemented in the library has both exportJSON and importJSON methods to allow easy access to this feature.

Other methods can broadly be categorised into the following categories:

\section{Tree manipulation methods}

These include functions to set the root based on a user-specified node or midpoint root, set the tree scale, and rotate nodes

\section{Tree appearance methods}

These include functions to collapse nodes, change the tree element styles (line width, node colour, node size and node shape), change the tree type (Circular, Diagonal, Hierarchical, Radial, or Rectangular) and set label font size. Tree leaves can also be annotated with metadata blocks to represent different attributes associated with the samples that the leaves represent.

\section{Tree interaction methods}

These include panning, zooming, tooltips, highlighting, and selection as demonstrated in this example: https://www.phylocanvas.gl/examples/08-interactive-tree.html.

Comprehensive documentation, code examples and code are available at https://phylocanvas.gl/.

\section{Worked examples}

To illustrate how the library can be used a few simple examples from the documentation are highlighted below

1. Simplest example where a newick tree string is rendered https://www.phylocanvas.gl/examples/\#basic

2. Fetch a newick tree from URL and set the node size and colour https://www.phylocanvas.gl/examples/fetch-tree-from-url.html

3. Fetch a metadata csv file which encodes colours and use these to change leaf node colours https://www.phylocanvas.gl/examples/fetch-tree-and-metadata-from-urls.html

4. Download tree as JSON including node properties https://www.phylocanvas.gl/examples/download-tree-as-json.html

5. Load tree properties from a JSON file and update on clicking a button https://www.phylocanvas.gl/examples/load-tree-props-from-json.html

6. Fetch a very large tree with 939298 leaves and colour based on a lineage encoded in the leaf labels 
k!-- (1) Load bundled version of Phylocanvas.gl library (https://www.phylocanvas.gl/docs/\#script-tag) --> kscript src="https://unpkg.com/ephylocanvas/phylocanvas.gl@latest/dist/bundle.min.js" $\rangle /$ script>

! -- (2) Create an empty DOM element to contain the tree $-->$

kiv id="demo" $>\langle/$ div $>$

(3) Load phylocanvas library $-->$

script>

// create a Phylocanvas.gl tree instance with a different style for each leaf node

const tree $=$ new phylocanvas. PhylocanvasGL (

// specify the DOM element in which the tree will be rendered

document. queryselector ("\#demo"),

\{

styles: \{

// set colour of node to red (default shape and label in newick string used)

Bovine: \{ fillcolour: "red" \},

// set shape to a star (default colour and label in newick string used)

Gibbon: \{ shape: phylocanvas.Shapes.Star \},

// set label (default colour and shape used)

Orangutan: \{ label: "Orangutan (Pongo pygmaeus)" \},

// hide label (default colour and shape used)

Gorilla: \{ label: " " \},

// set shape, colour, and label

Chimp: \{ shape: phylocanvas.Shapes.Hexagon, fillColour: "\#0000ff", label: "Chimpanzee" \},

// set colour as RGB in the format of $[x, g, b, a]$ (default shape and label as in Newick string)

Human: \{ shape: phylocanvas.Shapes.Triangle, fillColour: [ 0, 255, 0 ] \},

// set colour hsla (default label in newick string used)

Mouse: \{ shape: phylocanvas.Shapes.Dot, fillColour: "hsla (152, 100\%, 50\%, 1)" \}, \},

\section{// show leaf labels}

showlabels: true,

showleaflabels: true,

// set size of tree canvas (in pixels)

size: \{ width: 400, height: 300 \},

\section{// tree source as Newick string}

source: "(Bovine:0.69395, (Gibbon:0.36079, (Orangutan:0.33636, (Gorilla:0.17147," +

"(Chimp:1.19268, Human :0.11927) :0.08386) :0.06124):0.15057):0.54939, Mouse:1.21460);", \}, 
Phylocanvas.gl is utilised within https://microreact.org (as the tree rendering and interactivity engine and has been developed partly as a consequence of the scale-up required to enable trees produced from SARS-CoV-2 genomic initiatives such as the Covid-Genomics UK Consortium (COG-UK) to deliver interpretation of ever increasingly large trees (figure 1).

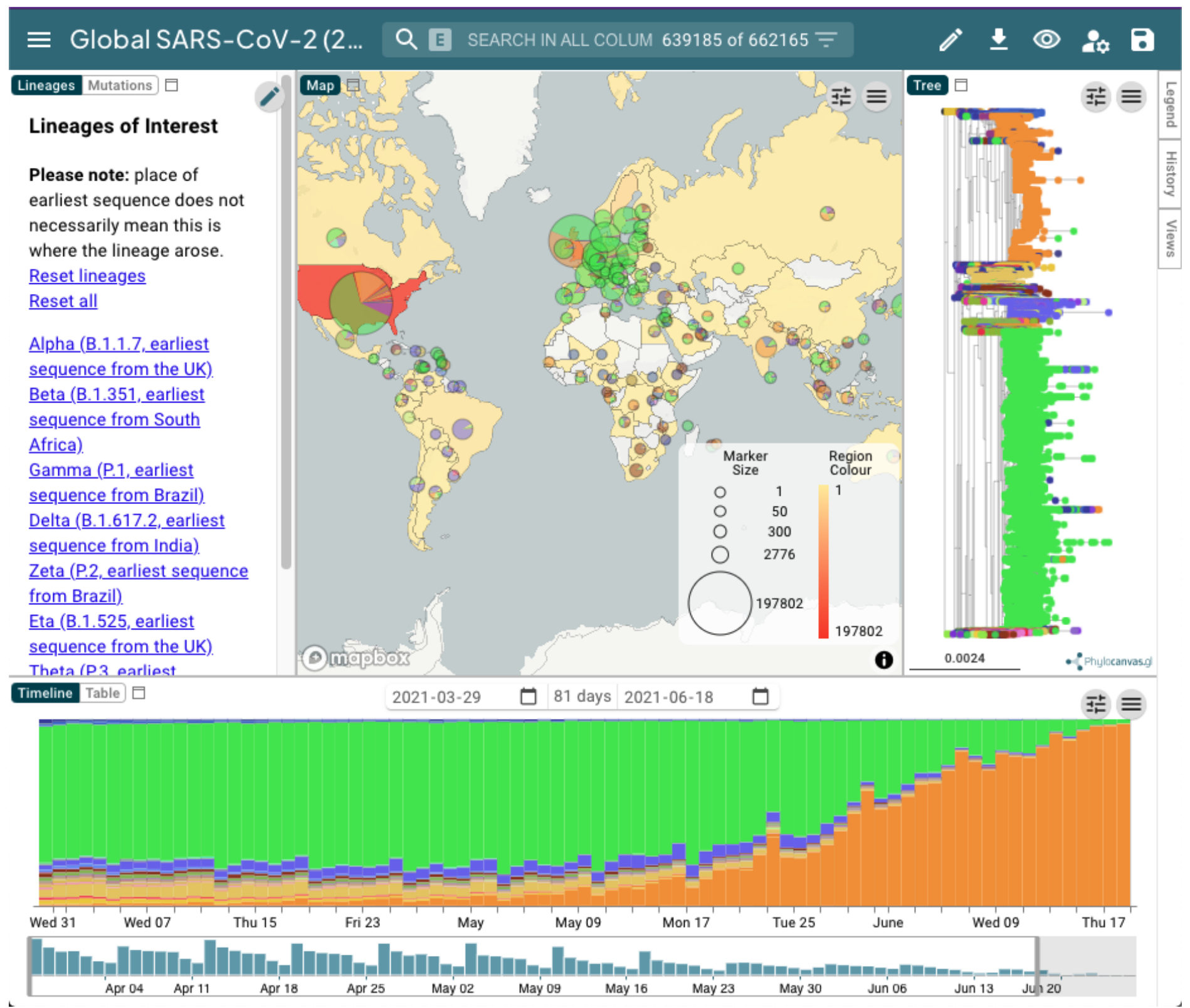

Figure 1: Integration of the phylocanvas.gl library into the application Microreact linking geographic, temporal and genomic data into a single view on a web page.

\section{Summary}

Phylocanvas.gl represents the next iteration of the Phylocanvas library, and provides an extensible and flexible library for utility within web applications. The library has a full feature set allowing configuration of many tree attributes and user interactivity. In addition, events within the rendered tree can be handled to allow developers to implement interaction with other application components. The library has the ability to render trees with in excess of hundreds of thousands of leaves as exemplified using SARS-CoV-2 data. 


\section{References}

1. phylotree.js - a JavaScript library for application development and interactive data visualization in phylogenetics https://doi.org/10.1186/s12859-018-2283-2

2. jsPhyloSVG: A Javascript Library for Visualizing Interactive and Vector-Based Phylogenetic Trees on the Web https://doi.org/10.1371/journal.pone.0012267

3. PhyD3: a phylogenetic tree viewer with extended phyloXML support for functional genomics data visualization https://doi.org/10.1093/bioinformatics/btx324

4. The Integrated Rapid Infectious Disease Analysis (IRIDA) Platform https://doi.org/10.1101/381830

5. CerealsDB 3.0: expansion of resources and data integration https://doi.org/10.1186/s12859-016-1139-x

6. Phandango: an interactive viewer for bacterial population genomics https://doi.org/10.1093/bioinformatics/btx610

7. COVID-19 Genomics Consortium UK - https://www.cogconsortium.uk/ 